\title{
Mitochondrial dysfunction and autism: comprehensive genetic analyses of children with autism and mtDNA deletion
}

\author{
Noémi Ágnes Varga ${ }^{1}$, Klára Pentelényi ${ }^{1}$, Péter Balicza ${ }^{1}$, András Gézsi ${ }^{1}{ }^{2}$, Viktória Reményi ${ }^{1}$, Vivien Hársfalvi ${ }^{1}$, \\ Renáta Bencsik', Anett Illés ${ }^{1}$, Csilla Prekop ${ }^{3}$ and Mária Judit Molnár ${ }^{{ }^{*}}$
}

\begin{abstract}
Background: The etiology of autism spectrum disorders (ASD) is very heterogeneous. Mitochondrial dysfunction has been described in ASD; however, primary mitochondrial disease has been genetically proven in a small subset of patients. The main goal of the present study was to investigate correlations between mitochondrial DNA (mtDNA) changes and alterations of genes associated with mtDNA maintenance or ASD.
\end{abstract}

Methods: Sixty patients with ASD and sixty healthy individuals were screened for common mtDNA mutations. Next generation sequencing was performed on patients with major mtDNA deletions (mtdel-ASD) using two gene panels to investigate nuclear genes that are associated with ASD or are responsible for mtDNA maintenance. Cohorts of healthy controls, ASD patients without mtDNA alterations, and patients with mitochondrial disorders (non-ASD) harbouring mtDNA deletions served as comparison groups.

Results: MtDNA deletions were confirmed in 16.6\% (10/60) of patients with ASD (mtdel-ASD). In 90\% of this mtdelASD children we found rare SNVs in ASD-associated genes (one of those was pathogenic). In the intergenomic panel of this cohort one likely pathogenic variant was present. In patients with mitochondrial disease in genes responsible for mtDNA maintenance pathogenic mutations and variants of uncertain significance (VUS) were detected more frequently than those found in patients from the mtdel-ASD or other comparison groups. In healthy controls and in patients without a mtDNA deletion, only VUS were detected in both panel.

Conclusions: MtDNA alterations are more common in patients with ASD than in control individuals. MtDNA deletions are not isolated genetic alterations found in ASD; they coexist either with other ASD-associated genetic risk factors or with alterations in genes responsible for intergenomic communication. These findings indicate that mitochondrial dysfunction is not rare in ASD. The occurring mtDNA deletions in ASD may be mostly a consequence of the alterations of the causative culprit genes for autism or genes responsible for mtDNA maintenance, or because of the harmful effect of environmental factors.

Keywords: Autism, Mitochondrial dysfunction, mtDNA deletion, ASD associated genetic alterations, Intergenomic communication

\section{Background}

In recent years, the number of patients diagnosed with autism spectrum disorders (ASD) has increased with

\footnotetext{
*Correspondence: molnar.mariajudit@med.semmelweis-univ.hu ${ }^{1}$ Institute of Genomic Medicine and Rare Disorders, Semmelweis University, Tömő Str. 25-29, Budapest 1083, Hungary

Full list of author information is available at the end of the article
}

current studies reporting a prevalence of $1 \%$ [1]. ASD shows extreme clinical heterogeneity; however, the diagnosis of ASD according to the Diagnostic and Statistical Manual of Mental Disorders (5th edition) is based on deficits in two areas-social communication and restricted, repetitive behaviour or interests. The patient must have deficits in both areas, and symptoms must be present from early childhood [2]. The genetic architecture of ASD 
is very diverse consisting of a variety of genetic alterations, such as chromosomal abnormalities, copy number variations, rare single nucleotide variants (SNVs), common polymorphic variations, and epigenetic modifications; however, only $6-15 \%$ of children with ASD have well-defined genetic syndromes [3]. Because of the development of high-throughput sequencing methods, many highly penetrant genetic causes of ASD have been identified, but the underlying genetic background of $70 \%$ of cases remains unexplained [4].

Mitochondrial disease (MD) is presently one of the most recognized metabolic diseases caused by the failure of both nuclear and/or mitochondrial DNA (mtDNA). The prevalence of mtDNA mutations responsible for MD is 1 in 5000 , whereas that of nuclear mutations is 2.9 per 100,000 cases [5]. Although MD frequently results in a spectrum of disorders with multisystemic presentations, neurological symptoms are common because tissues with high-energy demands, such as neural tissue, are often the most strongly affected by mitochondrial dysfunction. Even though the diagnosis of MD is increasing and becoming more frequent, the exact genetic background in many cases remains unconfirmed. Mitochondrial dysfunction can be caused by either primary MD or secondary mitochondrial damage [6]. Primary MD is because of genetic defects in mtDNA or a defect in a nuclear gene that is important for mitochondrial function. These mutations usually affect proteins involved in reactions of oxidative phosphorylation (OXPHOS). However, many disorders show similar effects in terms of mitochondrial dysfunction, but are elicited by mutations in other genes not related directly to normal mitochondrial function [7]. In other cases environmental factors, associated disorders or ageing are resulting in secondary alterations.

Several authors have proposed that mitochondrial dysfunction may be one of the most common medical conditions associated with autism $[8,9]$. Lombard et al. [10] proposed that ASD may be a condition with abnormal mitochondrial function. Clinical and biochemical studies have uncovered an emerging link between mitochondrial dysfunction and neurodevelopmental disorders, including intellectual disability [11], childhood epilepsy, and ASD [9]. Furthermore, mitochondrial dysfunction has been associated with some forms of syndromic ASD $[8,11]$. In many of these studies, biochemical changes, such as elevated levels of creatine kinase, lactate, pyruvate, carnitine, ammonia, and alanine were detected in the serum of patients with ASD [11-14]. In other studies, altered respiratory chain enzyme activities [15] or decreased expression of OXPHOS genes were detected in autistic brain [16], findings which indicate abnormal or altered mitochondrial function.
Damage to the OXPHOS system was found in individuals with ASD by Napoli et al. [17] and reviewed by Valenti et al. [11]. Oliveira et al. [14] found that 7\% (7/100) of children with ASD, who were clinically indistinguishable from other affected children with ASD, exhibited a mitochondrial respiratory chain disorder. Weissman et al. [18] proposed that defective mitochondrial OXPHOS may be an additional underlying pathogenic mechanism in a subset of individuals with autism.

Despite evidence of altered mitochondrial function in some individuals with ASD, it is not known whether mitochondrial dysfunction is a cause or an effect of ASD. Although a mitochondrial subgroup in ASD could be identified [19], findings from review articles, such as those of Palmieri and Persico [19] and Rossignol and Frye [9], found that even in this subgroup the causative genetic factor could be identified in a proportion of cases (23\%). In cases of non-syndromic ASD, mitochondrial dysfunction without mtDNA alterations has been frequently observed $[8,9]$. In a systematic review and metaanalysis, Rossignol reported that MD was present in 5\% of children with ASD [9], and in this ASD/MD subgroup, mtDNA abnormalities were found in $23 \%$ of patients [9]. These findings demonstrate that primary MD may be present in a subgroup of children with ASD.

Some studies have reported mtDNA deletions in individuals with ASD [12, 20-22]. Single mtDNA deletions have a role in different paediatric and adult onset primary MDs such as Kearns-Sayre syndrome, Pearson syndrome, and progressive ophthalmoplegia externa [23]. Multiple mtDNA deletions occur mostly because of pathogenic mutations in genes responsible for intergenomic communication; however, they are often related to ageing or harmful environmental factors as well because mtDNA has a poor DNA repair system [6, 24].

The aim of the present study was to investigate the presence of the most common pathogenic mtDNA alterations in patients with ASD and to elucidate the etiology of these mtDNA alterations by analysing their co-occurrence with both known ASD-associated genes and genes responsible for mtDNA maintenance and by comparison the targeted NGS data (ASD associated genes and genes responsible for mtDNA maintenance) of cases with and without mtdel-ASD, patients with primary mitochondrial disorders and healthy controls.

\section{Methods \\ Patients}

Detailed clinical examinations consisting of a general medical examination and neurological assessment were performed. A diagnosis of ASD was made using the ADI-R (autism diagnostic interview-revised) and ADOS 
(autism diagnostic observation schedule). Patients were screened for minor physical abnormalities, which were selected based on the Méhes Scale [25]. Family history and detailed environmental/societal data were collected from the first degree relatives of each patient. Any disorders present in the parents as well as environmental factors were registered. Written informed consent was obtained from the parents of the patient. This study was performed in accordance with the Helsinki Declaration of 1975 and was approved by the Hungarian Research Ethics Committee (44599-2/2013/EKU). The diagnosis of ASD was based on the standardized ADI-R in Hungarian, which was published by the Autism Foundation (Kapocs Publisher), according to the following scores: $\mathrm{A} \geq 10$ (social interaction), $\mathrm{B} \geq 7$ (communication), $\mathrm{C} \geq 3$ (repetitive stereotype manner), $\mathrm{D} \geq 1$ (abnormal development under 36 months). Sixty children with ASD [6 females and 54 males, median age $=7$ years, interquartile range $(\mathrm{IQR})=7.25]$ were included in our study. Before patient selection our ASD patients were screened for Fragile X syndrome and only negative cases were included in our cohort. Of our 60 patients with ASD, 58 are of European descent and 2 are Roma. Our control group for mtDNA screening consisted of 60 European adults (26 females and 34 males, median age $=28$ years, $\mathrm{IQR}=13.75$ ) selected from our biobank [26]. All controls were healthy individuals under 45 years of age and free from addiction (alcohol, smoking, and drugs). For the interpretation of our next generation sequencing (NGS) results, we compared data from the following cohorts: patients with ASD and without mtDNA deletion, labelled non-mtdel-ASD, (6 males and 1 female, median age $=8$ years, IQR $=5.5$ ), patients with MD and mtDNA deletion, without ASD (4 males and 3 females, median age $=18$ years, IQR $=19)$, and healthy control individuals ( 1 male and 5 females, median age $=27$ years, IQR $=2.25)$. The investigated patients and controls were not related. All patients without a mtDNA deletion were considered to have non-syndromic ASD. The study design is illustrated in Table 1.

\section{Genetic analysis}

DNA was isolated from peripheral blood samples from all participants using the QIAamp DNA blood kit (Qiagen, Hilden, Germany) according to manufacturer's instructions. To identify single and multiple mtDNA deletions, long range PCR was performed as described by Remenyi et al. [27]. MtDNA single and multiple deletions were screened with long PCR in $20 \mu \mathrm{l}$ volume: 20 pmol primers $\mathrm{Fw} 5^{\prime}$-TAAAAATCTTTGAAATAGGGC-3' ${ }^{\prime}$ and Rev 5'-CGgataCAGTTCACTTTAGCT-3', $0.2 \mu$ l Phusion DNA Polymerase (Finnzymes, Vantaa, Finland), $4 \mu \mathrm{l}$ Phusion GC Reaction Buffer (Finnzymes, Vantaa, Finland), $0.4 \mu \mathrm{l} \mathrm{dNTP}$ and $12.4 \mu \mathrm{l}$ water (qPCR grade water, AMBION). PCR program was the following: $98{ }^{\circ} \mathrm{C} 30 \mathrm{~s}$, 30 cycles: $98{ }^{\circ} \mathrm{C} 10 \mathrm{~s}, 63{ }^{\circ} \mathrm{C} 10 \mathrm{~s}, 72{ }^{\circ} \mathrm{C} 3 / 8 \mathrm{~min}$, then the last synthesis at $72{ }^{\circ} \mathrm{C} 7 \mathrm{~min}$. Amplificates were visualised by ethidium-bromide ( $2 \%$ agarose) and determined with QuantityOne Software (Bio-Rad Corp. Hertfordshire, UK). The three most-frequent pathogenic mtDNA point mutations were screened by PCR-RFLP using a GeneAmp PCR System 9700 (Applied Biosystems, MA, USA) [20]. The most well-known ASD-associated genes [28] and 51 genes responsible for intergenomic communication disturbances (Additional file 1: Table S1) were investigated using NGS, which was performed on a MiSeq (Illumina, CA, USA) using the TruSight Autism Rapid Capture Kit (Illumina, CA, USA) and the SureSelect QXT Kit (Agilent Technologies, CA, USA) according to the manufacturer's instructions. In the intergenomic panel, 16/32 samples were multiplexed in one sequencing run, whereas in the autism panel 24 samples were multiplexed in a single run using the MiSeq reagent kit v2 and 300 cycles (Illumina, CA, USA). The mean read depth was $152 \times$ in the intergenomic gene panel and $135 \times$ in the ASD-associated gene panel. In both panels, $20 \times$ coverage was achieved in a minimum of $90 \%$ of target regions. Pathogenic and likely pathogenic mutations from NGS data were validated by Sanger sequencing, and segregation analysis was performed within individual families.

Table 1 The design of the study

\begin{tabular}{llll}
\hline Cohorts & $\begin{array}{l}\text { M.3243 A }>\text { G, m.8993 T }>\text { C/G, } \\
\text { m.8344 A }>\text { G }\end{array}$ & mtDNA deletion & IG NGS (51 genes) \\
\hline ASD cases $(n=60)$ & $\checkmark$ & $\checkmark$ & $\checkmark$ ASD NGS (101 genes) \\
Healthy controls $(n=60)$ & $\checkmark$ & $\checkmark$ & $\checkmark b$ \\
mtdel-MD $(n=7)$ & $\checkmark$ & $\checkmark$ & $\checkmark$ \\
\hline
\end{tabular}

The investigated cohorts and the performed genetic analysis are shown in the Table 1. NGS testing for intergenomic panel and ASD panel has been performed in the cohort of the $10 \mathrm{mtdel}$ ASD cases and in subgroup of 7 non-mtdel ASD cases and a subgroup of healthy controls $(\mathrm{N}=6)$. Patients with primary mitochondrial disease $(\mathrm{N}=7)$ served as further control group. All investigated person were Caucasian except 2 non-mtdel ASD cases

ASD autism spectrum disorder, MD mitochondrial disease, $m$ tDNA mitochondrial DNA, IG NGS next generation sequencing for genes responsible for intergenomic communication, ASD NGS next generation sequencing for autism associated genes

a The 10 mtdel-ASD cases and 7 non-mtdel ASD were investigated

b 6 cases were investigated 


\section{Statistical and bioinformatics analysis}

Chi square test with Yates correction/Fisher exact test were used to determine significant differences between patient and control groups [29]. Raw sequences were filtered with Picard tools (version 2.1.0) [30] and quality filtered reads were aligned to the hg19 reference genome with BWA-mem [31] using default parameters. Variant calling was performed using GATK HaplotypeCaller (version 3.3-0) [32] and VCF files were annotated with SnpEff (version 4.1) [33]. We analysed only those variants that were found in the canonical transcript of the gene. To identify potentially causal genetic variations, we used VariantAnalyzer, which is an in-house software developed by András Gézsi from the Budapest University of Technology and Economics [34]. This software application annotates SNPs and short indels with several types of annotations, such as their predicted function on genes using SnpEff, observed allele frequencies in several genomic projects including the 1000 Genomes Project and the ESP6500 Project, conservation scores based on PhyloP or PhastCons, predicted function of non-synonymous SNPs using dbNSFP, and disease associations with HGMD and ClinVar. By creating filter cascades based on these annotations and other information (e.g., genotypes and variant quality annotations), the software can easily be used to filter the variants through a user-friendly graphical interface. Analysis and variant calling of SureSelect libraries was performed with SureCall software (Agilent, CA, USA). First, we filtered for variants known to be disease-causing, using human gene mutation database (HGMD) Professional 2015.1 edition [35]. Second, we filtered for rare variants based on the minor allele frequency and frequency of the mutation in our NGS data repository. Since large-scale genomic data of the Hungarian population is not available, a mutation with a low minor allele frequency may also be population-specific. We labelled a variant as a rare mutation if it was present in one or two samples within our cohort and the minor allele frequency in Europeans from the 1000 Genomes and ExAC databases was less than $0.5 \%$. It is important to note that a limitation of this method is that it may exclude identification of founder mutations and diseaseassociated polymorphisms. Finally, mutations were prioritized based on their predicted effects. Exonic frameshift and stop mutations were considered always damaging, whereas the effects of missense mutations were predicted using Polyphen2, SIFT (Sort Intolerant From Tolerant), or MutationTaster (MT).

\section{Results}

Sixty children with ASD ( 6 females and 54 males, median age $=7$ years, $\mathrm{IQR}=7.25$ ) were investigated. In our analysed cohort of 60 patients, 29 patients were sporadic (from simplex families) and their family histories were negative for other neurodevelopmental disorders and major psychiatric or neurological disorders. The distribution of the relevant symptoms for mitochondrial disorders in mtdel-ASD and idiopathic ASD group are shown in Fig. 1. There were some differences between ASD cases with and without mtDNA deletion regarding the clinical phenotype. Developmental regression, muscle hypotonia, and additional neurological signs were most common in the mtdel-ASD cases. Multisystemic abnormality appeared also more frequently. Referring to seizures no major differences has been observed between these two groups. Family history was not available in two cases because these children were not living with their biological parents. A positive family history was found in $48 \%$ of cases, of which 20 cases $(33.3 \%)$ had a family history for psychiatric disorders (bipolar disorder, depression, and schizophrenia). In four cases (6.7\%), visual and hearing impairments, ataxia, complex endocrine disorders, or a combination of these factors was noted. The co-occurrence of these symptoms is an indicator for MD according to mitochondrial disease criteria (MDC) [36]. In five cases (8\%), we found a positive family history for psychiatric disorders and some MDC-related symptoms were also noted.

Minor physical anomalies were identified in 44 children. All children were diagnosed with ASD based on ADI-R, and in most cases, with ADOS as well. We found that serum lactate levels and/or the lactate:pyruvate ratio supported the presence of mitochondrial dysfunction in four patients.

\section{Genetic investigation-mtDNA mutation screening}

Mitochondrial deletions were identified in 16.6\% (10/60) of our patients with ASD. Two children had multiple deletions, whereas a single major deletion was detected in the range of 2.4-7.9 kb in eight children. Detailed clinical and family history data as well as associated phenotype of the patients harbouring mtDNA deletions are shown in Table 2. An evaluation of clinical phenotype, family history, and laboratory data suggested MD in seven cases with mtDNA deletion. None of the investigated families had a previous diagnosis of primary MD. In all cases, the rate of heteroplasmy (HP) was $>20 \%$ in blood samples (Table 2). In the 60 healthy control individuals, a mtDNA deletion was found in two cases. Based on our statistical analysis, there was a significant difference in the frequency of mtDNA deletions between our ASD and control cohorts $\left(\mathrm{x}^{2}\right.$ with Yates correction $=4.5 ; \mathrm{p}=0.03$; odds ratio $=5.8 ; 95 \%$ CI 1.21-27.72). Further analysis of mtDNA mutational "hotspot" regions (m.3243 A > G, $\mathrm{m} .8993 \mathrm{~T}>\mathrm{C} / \mathrm{G}$, and $\mathrm{m} .8344 \mathrm{~A}>\mathrm{G}$ ) did not detect any alterations in our ASD cohort. 


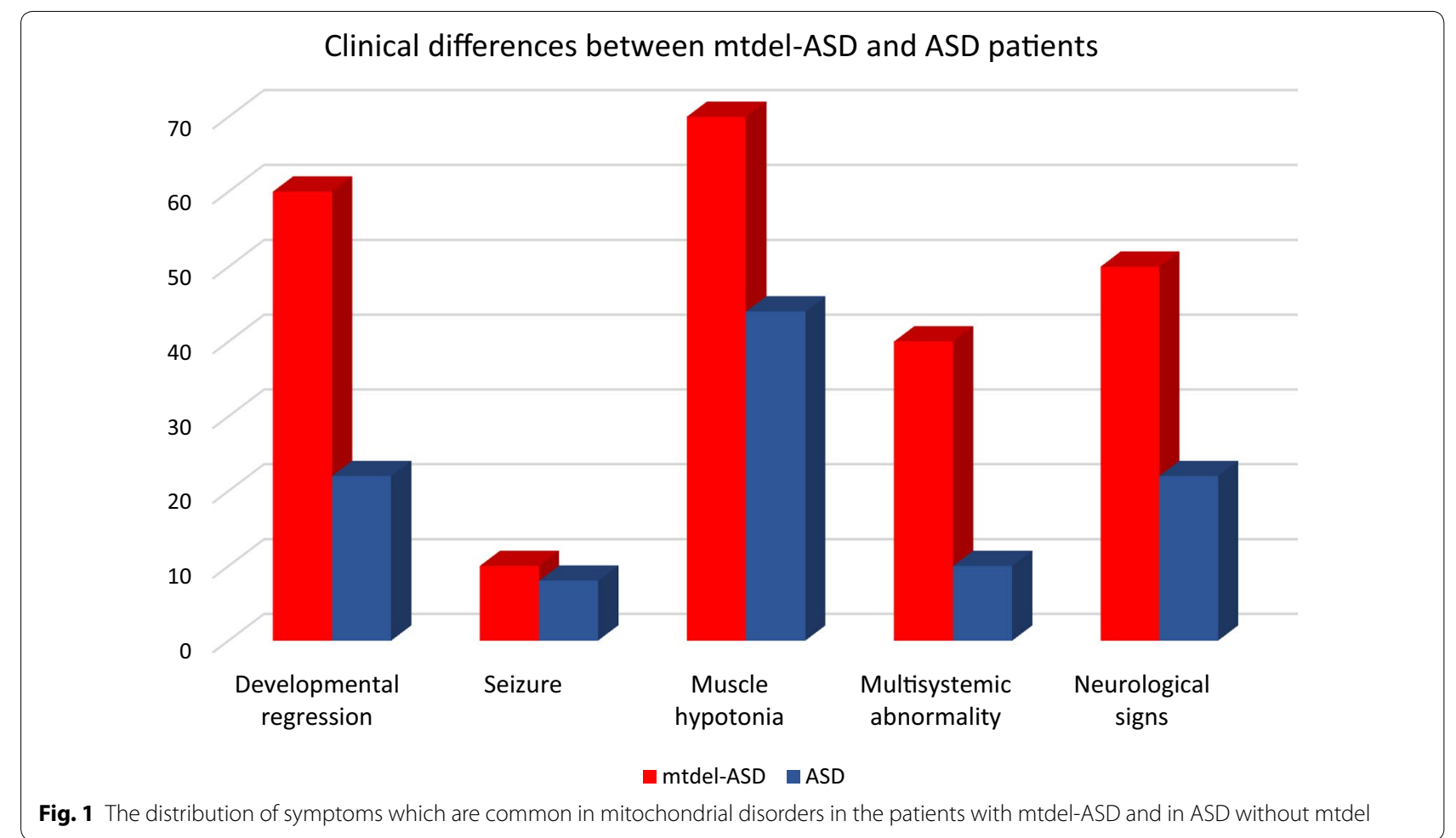

\section{Genetic investigation-nuclear DNA mutation screening} of genes responsible for intergenomic communication Next, we focused on those patients with mtDNA deletions (mtdel-ASD) and performed nuclear DNA (nDNA) mutation screening to investigate genes involved in intergenomic communication. In this subgroup, we found one rare likely pathogenic variant and one variant with uncertain significance (VUS). The rare variants identified in two patients were both present in only one allele, however the mode of inheritance of these disorders is autosomal recessive (Table 3). In Patient 5 (P5), the likely pathogenic heterozygous T265I mutation in the mitochondrial genome maintenance exonuclease 1 (MGME1) is responsible for mtDNA integrity [37]. In Patient 9 (P9), we found a de novo VUS mutation (ClinVar ID203970) in succinate-CoA ligase alpha subunit (SUCLG1) in a heterozygous form.

In our cohort of seven patients with MD (without ASD) harbouring mtDNA deletions, we found a pathogenic rare variants in two case, and rare VUS in five further cases (Table 2). In one patient the compound heterozygous state of one pathogenic and one VUS in C10orf2 gene were detected. In cohorts without MD (patients with ASD lacking mtDNA deletion and healthy individuals) we found two-two rare VUS in genes responsible for intergenomic communications (Table 3).
Comparing the intergenomic NGS panel results for our different cohorts, we found no significant difference between mtdel-ASD cases and healthy controls with one tailed Fisher exact test (p: 0.4890 odds: 0.5, CI 0.054.97); and mtdel-ASD and ASD without mtDNA deletion groups (p:0.55882, Odds: 0625 CI 0.06-5.96). Likely pathogenic variant and VUS were identified in higher number in MD patients with mtDNA deletion and without ASD ( $\mathrm{p}=0.013$, Odds: 0.04 CI 0.003-0.5743), in a heterozygous form (Table 3 ).

\section{Genetic investigation-nDNA mutation screening of ASD-associated genes}

Using the TruSight Autism NGS panel, we detected rare SNVs in 90\% (9/10) of our affected children with mtDNA deletion. Syndromic ASD was identified in a single case, Patient 3 (P3), from our mtdel-ASD cohort. A heterozygous pathogenic mutation in chromodomain helicase DNA-binding protein 7 (CHD7) was found in this patient as well as a heterozygous mutation of uncertain significance in tuberin (TSC2). The CHD7 rare variant regarding the ACMG guideline, fulfils the PVS1 and one PS2 criteria [38] and based on this we evaluated it as pathogenic. The patient's phenotype and the family segregation pattern indicated this $C H D 7$ mutation as a de novo mutation resulting in CHARGE syndrome (OMIM 214800) [39]. 


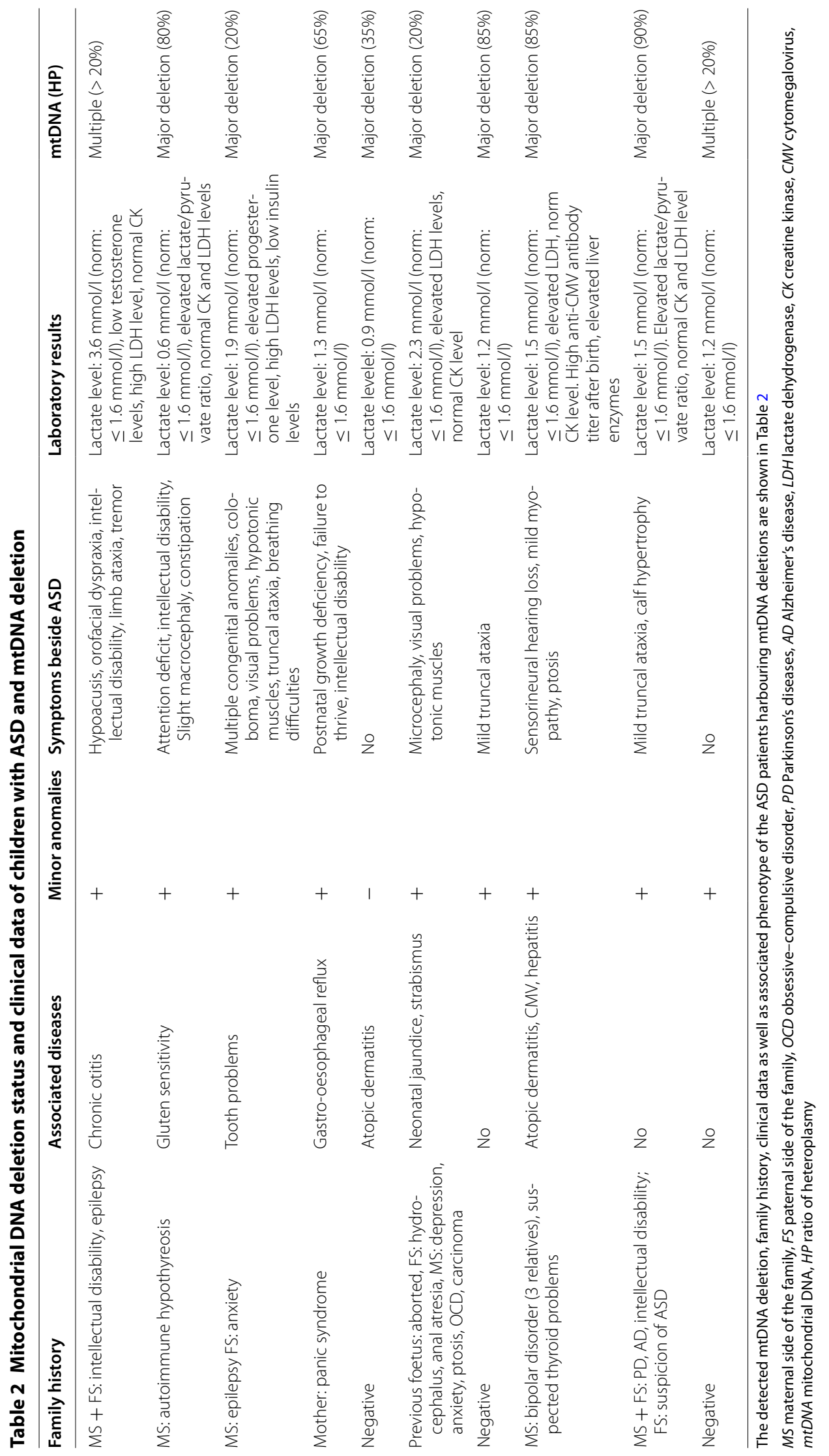




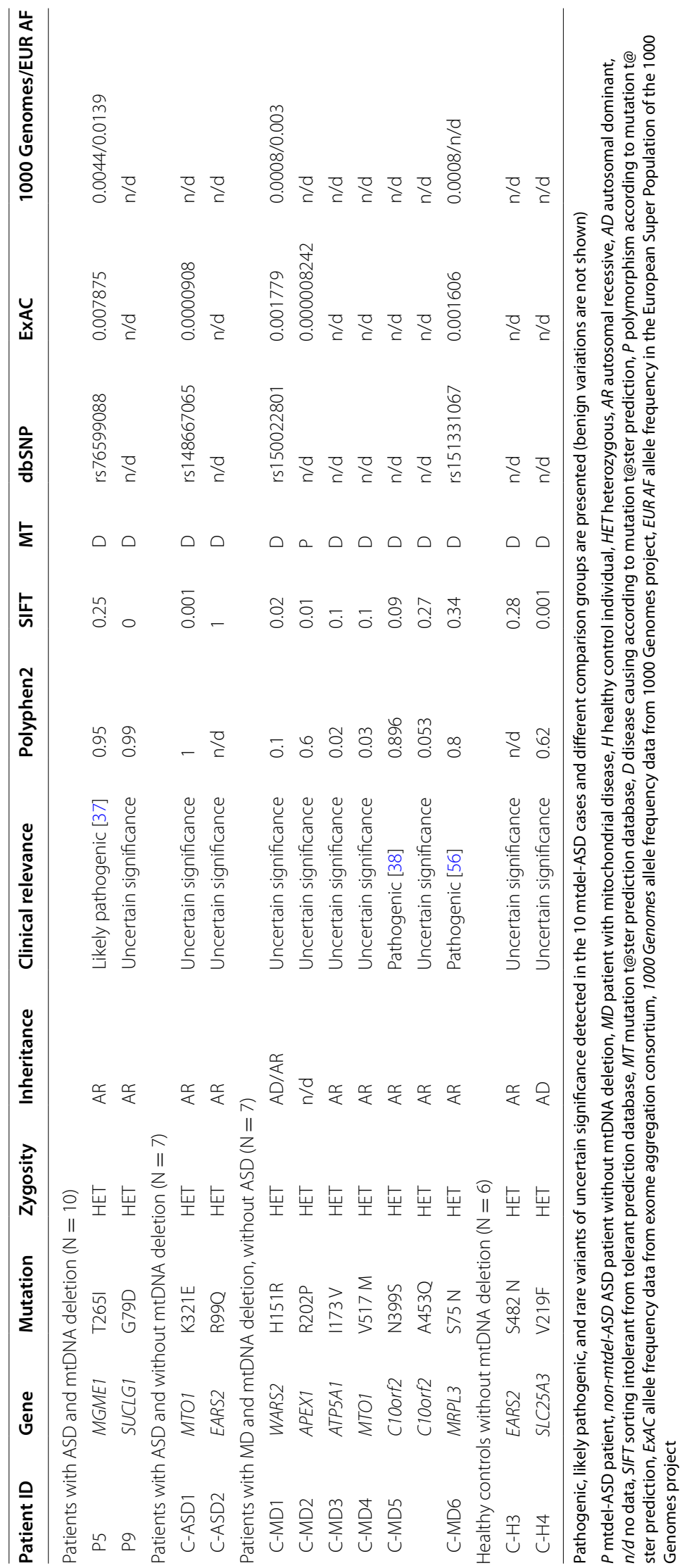


In Patient 8 (P8), we found a pathogenic nonsense mutation in 7-dehydrocholesterol reductase (DHCR7), which was present in only one allele.

In Patient 2 (P2) a rare mutation was detected in autism susceptibility candidate 2 (AUTS2), which previously was associated with syndromic ASD form. The significance of the missense mutation identified in our study is uncertain; during segregation analysis the same mutation was present in the healthy mother, however we do not know exactly the penetrance of the genetic defects of AUTS2. This rare AUTS2 variant coexisted with a rare variant in retinoic acid induced gene 1 (RAI1) (Table 4).

Using in silico analysis, alterations of uncertain significance were detected in ASD-associated genes in 60\% $(6 / 10)$ of the mtdel-ASD cases and 71\% (5/7) of nonmtdel-ASD cases (Table 4). In the six control individuals only four rare VUS were detected in genes associated with ASD. The rare variant in zinc finger protein $804 \mathrm{~A}$ (ZNF804A) was found in two healthy controls, indicating a variant that is likely population-specific (Table 4).

\section{Detailed phenotype of a patient with mtDNA deletion and CHARGE syndrome (CHD7 mutation)}

An 11-year-old male patient (P3) had multiple congenital anomalies, such as coloboma of the eyes, oxycephaly, epicanthus, convergent strabismus, mild bifid nose/ broad nasal tip, low settled cup ears, mild facial asymmetry, dental dysgenesis, asymmetric chest, macroglossia, cryptorchidism, testicular hypoplasia, and atrial septal defect. He began walking at 3 years of age and suffers from obsessive hand movements, erratic behavior, and sleep disturbance. Aside from his developmental abnormalities, neurological investigation detected pes varus, severe visual impairment, bilateral ptosis, chewing difficulties, mild atrophy and weakness in the distal muscles of the extremities, and truncal ataxia. High lactate levels were detected in both serum and cerebrospinal fluid, and decreased levels of serum melatonin, calcium, and vitamin D3 were measured. A brain MRI detected hypoplastic vermis and transverse sinus on the right side. An EEG found generalized irritative signs, and VEP found an increased P100 on the left side. Brainstem auditory evoked potentials was normal. Family history identified arrhythmia, diabetes mellitus, and colon polypomatosis on the maternal side, and diabetes mellitus, arrhythmia, and dementia on the paternal side.

\section{Detailed phenotype of a patient with congenital cytomegalovirus infection and mtDNA deletion}

The 5-year-old female patient (P8) was born at a gestational age of 39 weeks by Caesarean section with a birth weight of $3000 \mathrm{~g}$. The pregnancy was complicated with a partial placental abruption at 11 weeks. Evidence of prolonged neonatal jaundice, highly elevated liver enzymes, low prothrombin level, and high IgM and IgG type anti-cytomegalovirus (CMV) antibodies led to the diagnosis of a congenital CMV infection-induced hepatic lesion. She had congenital sensorineural hearing loss, mild myopathic facies, mild ptosis, and atopic facial dermatitis. A brain MRI identified several T2 hyperintense supratentorial lesions $(5-10 \mathrm{~mm}$ in size), which were suggested to have an infectious etiology. A heterozygous nonsense mutation in DHCR7 and a major large single deletion in mtDNA were found. The healthy mother also harbours the detected heterozygous mutation. Cholesterol and 7-dehydrocholesterol levels of the child are in the normal range. Homozygous or compound heterozygous mutations in DHCR7 result in Smith-Lemli-Opitz (SLOS) syndrome, which is an autosomal recessive disease. However, human CMV infection may lead to altered mitochondrial biogenesis [40]. We believe that this case demonstrates a direct interaction between genetic and environmental risk factors in some forms of ASD.

\section{Discussion}

In this study, we provide for the first time a comprehensive genetic analysis of patients with ASD that investigates co-occurrence of the most frequent mtDNA alterations, intergenomic communication disturbances (51 genes), and 101 genes previously associated with ASD. We found co-occurrence of mtDNA deletions with ASD-associated genetic alterations, which supports the previous observation that mitochondrial alterations are frequently associated with ASD. In one patient with ASD (P3), we found a mtDNA deletion with CHARGE syndrome caused by a de novo mutation in CHD7. These genetic alterations in P3 were also accompanied by a TSC2 mutation of uncertain significance. Autistic symptoms are present in approximately $30 \%$ of patients with CHARGE syndrome [39], and lactic acidosis is a rare alteration. Based on the phenotype, we conclude that the driving genetic alteration in this patient is the CHD7 mutation, and the mitochondrial gene defect may not be the true causative factor in the etiology of the disease; however, CHD7 function is strongly ATP-dependent [41]. In addition, the associated heterozygous TSC2 mutation is likely a modifying gene. $\mathrm{CHD} 7$ is a member of the chromo-domain helicase DNA-binding (CHD) protein family and plays a role in transcription regulation through chromatin remodelling. Mutations in TSC2 are known to cause one syndromic form of ASD; however, our patient did not develop the classic symptoms of tuberous sclerosis until recently. TSC2 mutations may induce activation of mTORC1 leading to increased mtDNA expression and mitochondrial density. mTOR is a Ser/Thr kinase that forms complexes with numerous 


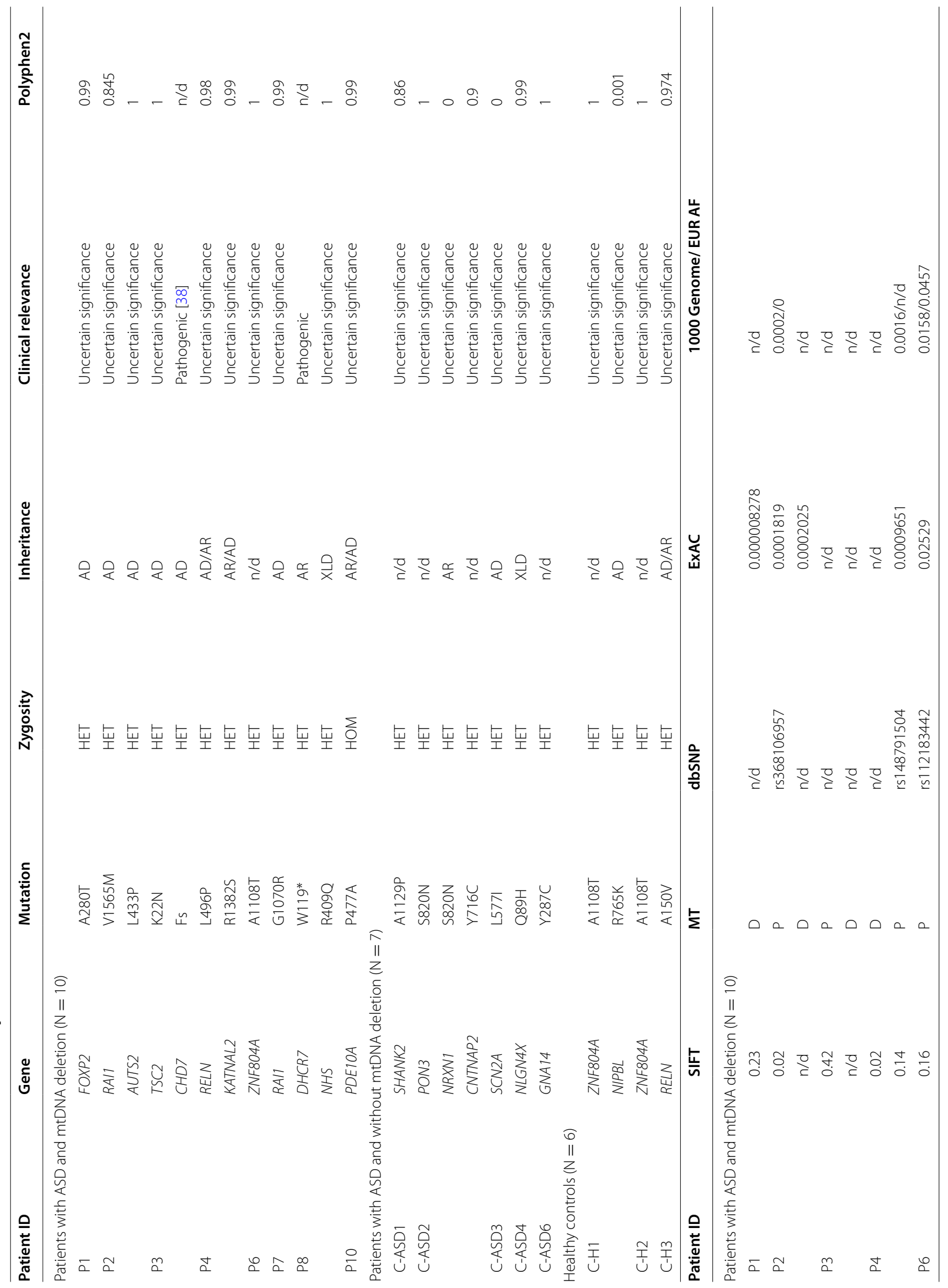


Varga et al. Behav Brain Funct (2018) 14:4

Page 10 of 14

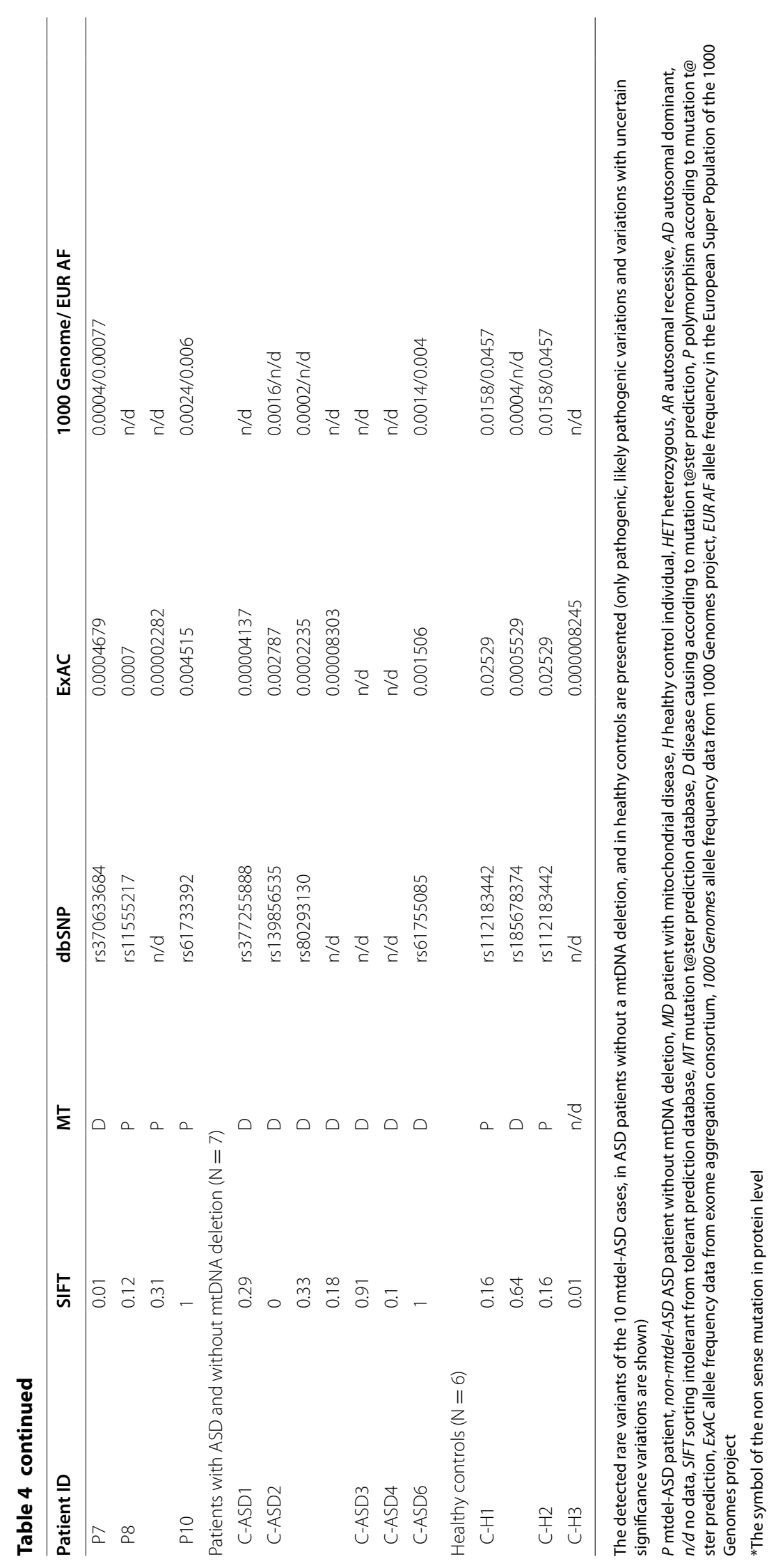


protein partners to regulate cell growth, mitochondrial membrane potential, and ATP synthetic capacity [42].

In Patient 8, we found that the mtDNA deletion was accompanied by a heterozygous pathogenic DHCR7 mutation and a rare variant of uncertain significance in NHS actin remodelling activator (NHS). DHCR7 catalyses cholesterol production from 7-dehydrocholesterol, and defects in this protein cause SLOS. Furthermore, a high 7-dehydrocholesterol level results in mitochondrial dysfunction [43]. However, the significance of a heterozygous mutation in this gene is not known. We hypothesize that in the case presented here the co-occurrence of the DHCR7 heterozygous mutation and CMV infection may play a role in changes of mitochondrial biogenesis and in the pathogenesis of autistic features. The patient was tested for SLOS; both serum cholesterol and 7-dehydrocholesterol levels were normal, which rules out the presence of typical SLOS.

Evidence of mitochondrial dysfunction in ASD was first described 19 years ago [10]. Currently, it is the most common metabolic abnormality known in ASD with a prevalence of $7.2 \%$ [14]. In a subgroup of the CHARGE (Childhood Autism Risk from Genes and Environment) study, decreased NADH activity was found in lymphocytes in 8 of 10 cases. In this cohort, only 2 of the 10 patients had mtDNA deletions and 5 patients had altered mtDNA copy numbers [12]. However, the genetic background was not clarified in $79 \%$ of the patients with ASD-MD [9]. Therefore, the possibility of secondary damage to mitochondria cannot be excluded. A small pilot study examining 12 patients with ASD described 8 mitochondrial deletions [21], which could be the result of intergenomic communication disturbances, environmental factors, or other gene-gene interactions. As is the case for many other disorders, it is still not clear whether the detected mitochondrial dysfunction in ASD is a primary or secondary event either having a key role in disease pathogenesis or is simply a downstream effect.

In our study, mtDNA deletions were identified in 16.6\% of evaluated patients with ASD. During mtDNA hotspot screening and NGS analysis, no concomitant primary MD was detected. To examine whether mtDNA deletion is a primary or secondary event in ASD in our cohort, we used different comparison groups to screen the nDNA background of the mtDNA deletion. Pathogenic or likely pathogenic variants were detected in both mtdel-ASD and MD without ASD cases, all in heterozygous form. A high number of VUS in intergenomic communication genes were detected in the MD without ASD cohort $(4 / 6)$, and a few rare variants were identified in patients with ASD that lacked mtDNA deletion and in healthy controls (Table 3). Homozygous or compound heterozygous mutations in MGME1 and SUCLG1 have been previously correlated with severe early-onset mitochondrial disorders (OMIM 615084, OMIM 245400) [44]. The importance of the presence of heterozygous mutations is not well understood. It is known in some genes responsible for intergenomic communications heterozygous mutations may result in a less severe phenotype than that found with the homozygous form [45].

The question has also been raised whether patients with MD and ASD symptoms have special characteristics. In a study by Rossignol and Frye [9], a cohort of ASD/MD children were compared to two comparison groups: children with general ASD and children with general MD. In the ASD/MD group, increased lactate and pyruvate levels, seizures, motor delays, and gastrointestinal abnormalities were significantly more prevalent compared to children with general ASD. A more balanced male:female ratio was also detected in the ASD/MD group [9]. Our results confirm the observations by Rossignol and Frye; however, in our ASD cohort with mtDNA deletion, elevated lactate levels and/or an elevated lactate:pyruvate ratio were found in only four cases, whereas most of our ASD patients with mtDNA deletion had symptoms common to MD, such as hypoacusis, muscle weakness, hypotonia, delayed motor development, and movement disorder (Fig. 1). Significant difference between mtdel-ASD and non-mtdel-ASD group was found regarding clinical phenotypes (developmental regression, muscle hypotonia, additional neurological signs and multisystemic alterations were more common in cases mt-delASD). Interestingly, the phenotypes of classic mitochondrial deletion syndromes, such as Pearson syndrome, progressive ophthalmoplegia externa, and Kearns-Sayre syndrome, were not detected in any of our patients. The family histories of mtdel-ASD children in our cohort differed from the family histories of the ASD cohort without a mtDNA deletion, since various psychiatric disorders were common among family members of mtdel-ASD cases both on maternal and paternal side. However none of the parents reached the MDC scoring cut-off value for definitive MD, which could not be independently verified because none of the family members agreed to perform muscle biopsy. MtDNA disorders are usually inherited maternally, however single mtDNA deletions are considered sporadic events with low inheritance risk, whereas multiple mtDNA deletions are the result of primary nuclear defects in genes responsible for mtDNA maintenance or nucleoside metabolism and follow Mendelian inheritance patterns [46].

Mitochondrial haplogroups were also investigated in association with ASD. Chalkia et al. found that individuals with European haplogroups designated I, J, K, X, T and $U$ ( $55 \%$ of the European population) had significantly higher risks of ASD compared to the most common European haplogroup, HHV. Asian and Native American haplogroups A and $\mathrm{M}$ also were at increased risk of ASD 
[47]. In Hungary it is not rare that a person has ancient European haplotype such as T, K, and U haplotype, and rarely Asian haplotype such as B can occur as well. In some Hungarian patients the mtDNA deletion was coexisting with ancient haplotype [48].

In $90 \%(9 / 10)$ of children from the mtdel-ASD cohort, we found rare SNVs in ASD-associated genes (Table 4). A rare mutation was detected in AUTS2 in which deletions are inherited in an autosomal dominant manner and are associated with neurological symptoms including intellectual disability and developmental delay [49]. In a modest study of 13 cases of ASD associated with AUTS2 alterations, only one patient had a nonsense mutation; all the other patients had a deletion [49]. The significance of the missense mutation identified in our study is uncertain (her mother harbours the mutation as well); however, clinical symptoms of the patient correlate with the phenotype of previously published AUTS2 mutations. This rare AUTS2 variant coexisted with a rare variant in retinoic acid induced gene 1 (RAI1). The gene-gene interaction of these two alterations are hypothesized.

In addition, we found that patients had mutations of uncertain significance in forkhead box P2 (FOXP2), RAI1, phosphodiesterase 10A (PDE10A), katanin catalytic subunit A1 like 2 (KATNAL2), and reelin (RELN). Most of these genes play a role in cell regulation, signal transduction, and various signalling pathways, which could influence mitochondrial function. FOXP2 is an evolutionarily conserved transcription factor that regulates the expression of a variety of genes. Mutations in this gene cause speech-language disorder 1 (OMIM 602081), which is also known as autosomal dominant speech and language disorder with orofacial dyspraxia [50]. RAI1 acts as a transcriptional regulator of chromatin remodelling by interacting with basic transcriptional machinery [51]. RAI1 deletion is associated with Smith-Magenis syndrome, whereas duplications are associated with Potocki-Lupski syndrome [52]. Several heterozygous mutations are also associated with Smith-Magenis syndrome [53]. In our case, we found that the typical symptoms of Smith-Magenis syndrome were not present. Mutations in PDE10A can affect cyclic nucleotide concentrations. This phosphodiesterase selectively catalyzes the hydrolysis of $3^{\prime}$ cyclic phosphate bonds in cAMP and/ or cGMP. The phosphodiesterase family of proteins regulates cellular levels, localization, and duration of action of these second messengers by controlling the rate of their degradation. In addition, phosphodiesterases are involved in many signal transduction pathways and are implicated in the pathogenesis of bipolar disorder [54].

Mitochondrial dysfunction may be associated with several forms of syndromic ASD, but is also frequently related to non-syndromic cases $[8,9]$. During our comprehensive analysis, we found examples of both but in most cases we did not find the causative genetic mutation that accounts for the mitochondrial dysfunction. In the examined children from the general ASD cohort (without mtDNA deletion), we found several VUS, most of which were identified in genes without previous correlation to mitochondrial dysfunction. Based on our findings, we conclude that the detected mitochondrial DNA deletions in patients with ASD in our cohort are a secondary effect. By investigating the most common mtDNA alterations and the most common nuclear genes responsible for intergenomic communications, we did not identify the clear genetic etiology in most of our cases. Therefore, further investigation and characterization is warranted.

\section{Limitations}

We identified certain limitations in our study. We focused our investigation to analyse mutational hotspots and large mtDNA deletions and did not sequence the entire mitochondrial genome. The mtDNA mutations were analysed from blood samples; postmitotic tissue was not available. The used long PCR method detects deletions in the mtDNA with high sensitivity and low specificity. Deletions under $10 \%$ of heteroplasmy could be missed due to technical barrier, overestimation of the HP ratio is not expected. The detection of mtDNA deletion from NGS data will be in the future a new perspective, but today it is not in the everyday praxis. We used targeted NGS panels comprised of the most important genes associated with intergenomic communication and ASD. However, these panels do not include all currently associated genes and the number of these genes is continuously increasing. Finally, our healthy control group was older than our ASD cohort and had different gender ratios. However, we felt that ethically it was not appropriate to obtain biomaterial from healthy children for genetic testing. Since somatic mtDNA deletion may occur in association with ageing, and we detected the mtDNA deletion less frequently in the control group it had no impact on our data. Mitobreak Database [55] supports or presumption since deletion were present mostly only aged healthy controls, otherwise they were associated or to sporadic primary mitochondrial disorders (single deletion) or to disorders due to intergenomial gene alterations (multiple deletions).

\section{Conclusions}

The aim of our study was to gain a better understanding of mitochondrial dysfunction in autism. We found that mtDNA alterations were more common among our cohort of patients with ASD than in control individuals. In addition, we found that the mtDNA deletion was 
usually not the single genetic alteration identified in ASD, but co-occurred in both syndromic and non-syndromic forms of ASD with either ASD-associated genetic risk factors and/or alterations in genes responsible for intergenomic communication. Our findings indicate a very complex pathophysiology of ASD in which mitochondrial dysfunction is not rare and can be caused by mtDNA deletion, which may be considered as de novo mutations or the consequence of the alterations of the causative culprit genes for autism or genes responsible for mtDNA maintenance.

\section{Additional file}

Additional file 1: Table S1. Investigated genes responsible for mtDNA maintenance (intergenomic NGS panel).

\section{Abbreviations}

ACMG: American College Medical Genetics; AD: autosomal dominant inheritance; AD: Alzheimer's disease; ADI-R: autism diagnostic interview_revised; ADOS: autism diagnostic observation schedule; AR: autosomal recessive inheritance; ASD: autism spectrum disorder; ASD NGS: next generation sequencing for autism associated genes; ATP: adenosine-triphosphate; BWA: BurrowsWheeler alignment tool; C-ASD: control ASD patient; C-H: healthy control individual; CHARGE: coloboma, heart defect, atresia choanae, retarded growth and development, genital-, ear abnormality; CHARGE: childhood autism risk from genes and environment; CHD: chromo-domain helicase DNA-binding; CK: creatine kinase; C-MD: control patient with MD; CMV: cytomegalovirus; $\mathrm{D}$ : disease-causing according to mutation t@ster prediction; dbNSFP: database for nonsynonymous SNPs'functional predictions; DNA: deoxyribonucleic acid; EEG: electroencephalography; ESP6500: NHLBI GO exome sequencing project; EUR AF: Allele frequency in the European super population of the 1000 Genomes project; ExAC: exome aggregation consortium; FS: on father's side; Fs: frameshift mutation; FS: father's side of the family; HET: heterozygous; HGMD: human gene mutation database; HOM: homozygous; HP: heteroplasmy; IG NGS: next generation sequencing for genes responsible for intergenomic communication; IQR: interquartile range; LDH: lactate dehydrogenase; MD: mitochondrial disease; MDC: mitochondrial disease criteria; MRI: magnetic resonance imaging; MS: on mother's side; MS: mother's side of the family; MT: mutation taster prediction; mtdel-ASD: ASD patients with mitochondrial deletion; mtDNA: mitochondrial deoxyribonucleic acid; mTORC: mechanistic target of rapamycin complex; n/d: no data; NADH: nicotinamide adenine dinucleotide; nDNA: nuclear deoxyribonucleic acid; NGS: next generation sequencing; OCD: obsessive-compulsive disease; OXPHOS: oxidative phosphorylation; P: polymorphism according to mutation t@ster prediction; P1-10: mtdel-ASD patient 1-10; PCR: polymerase chain reaction; PD: Parkinson's disease; PS2: pathogenic criterion is weighted as strong; PVS1: pathogenic criterion is weighted as very strong; RFLP: restriction fragment length polymorphism; SIFT: sort intolerant from tolerant amino acid substitution prediction software; SLOS: Smith-Lemli-Opitz syndrome; SNV: single nucleotide variant; VCF: variant call format; VEP: visual evoked potential; VUS: variant of uncertain significance; XLD: X-linked dominant inheritance.

\section{Authors' contributions}

MMJ, NÁV and KP designed the study. NÁV and PB performed data collection, physical examination of the patients. KP and VH performed the library preparation for NGS panels, NÁV analyzed the NGS data of the autism panel, and control groups, KP analyzed the NGS data of the intergenomic panel, and participated in mtDNA mutation screening and manuscript drafting. AG provided the bioinformatical platform to analyze the NGS data, VR participated in mtDNA deletion screening, $\mathrm{VH}$ preformed NGS sequencing. $\mathrm{RB}$ made the validation by Sanger sequencing and the segregation analysis of the family members. Al revised the manuscript. CsP performed the neuropsychological testing of the patients, NÁV wrote the manuscript and performed the statistical analyses. MJM designed the study, coordinated the research team, leaded the manuscript preparation, revised and corrected the manuscript. All authors read and approved the final manuscript.

\section{Author details}

${ }^{1}$ Institute of Genomic Medicine and Rare Disorders, Semmelweis University, Tömő Str. 25-29, Budapest 1083, Hungary. ${ }^{2}$ Department of Genetics, Celland Immunobiology, Semmelweis University, Nagyvárad tér 4, Budapest 1089, Hungary. ${ }^{3}$ Vadaskert Foundation for Children's Mental Health, Lipótmezei Str. 1-5, Budapest 1021, Hungary.

\section{Acknowledgements}

The authors thank Margit Kovács, Mariann Markó, and Mónika Sáry for their technical assistance, Lisa Hubers for language corrections, and the Metabolic Laboratory of the I. Department of Pediatrics for performing the biochemical investigation.

\section{Competing interests}

The authors declare that they have no competing interests.

\section{Availability of data and materials}

All the datasets and/or analyses are available for reasonable from authors.

\section{Consent for publication}

All of the authors have agreed to publish this manuscript.

\section{Ethics approval and consent to participate}

The Hungarian Research Ethics Committee approved the study. Approval Number is: 44599-2/2013/EKU (535/2013). The patient's parents gave written informed consent

\section{Funding}

This study was supported from Research and Technology Innovation Fund BIOKLIMA KTIA_AIK_12-1-2013-0017 and Hungarian National Brain Research Program KTIA_NAP_13_1-2013-0001.

\section{Publisher's Note}

Springer Nature remains neutral with regard to jurisdictional claims in published maps and institutional affiliations.

Received: 24 August 2017 Accepted: 16 January 2018

Published online: 20 February 2018

\section{References}

1. Lai MC, Lombardo MV, Baron-Cohen S. Autism. Lancet. 2014;383(9920):896-910.

2. American Psychiatric Association. Diagnostic and statistical manual of mental disorders: DSM-5-. 5th ed. Washington, DC: American Psychiatric Association; 2013. p. 50.

3. Schaefer GB, Mendelsohn NJ. Genetics evaluation for the etiologic diagnosis of autism spectrum disorders. Genet Med. 2008;10(1):4-12.

4. Schaaf $C P$, Zoghbi HY. Solving the autism puzzle a few pieces at a time. Neuron. 2011;70(5):806-8.

5. Gorman GS, Schaefer AM, Ng Y, Gomez N, Blakely EL, Alston CL, et al. Prevalence of nuclear and mitochondrial DNA mutations related to adult mitochondrial disease. Ann Neurol. 2015;7(5):753-9.

6. Niyazov DM, Kahler SG, Frye RE. Primary mitochondrial disease and secondary mitochondrial dysfunction: importance of distinction for diagnosis and treatment. Mol Syndromol. 2016;7(3):122-37.

7. Martikainen MH, Chinnery PF. Mitochondrial disease: mimics and chameleons. Pract Neurol. 2015;15(6):424-35.

8. Frye RE, Rossignol DA. Mitochondrial dysfunction can connect the diverse medical symptoms associated with autism spectrum disorders. Pediatr Res. 2011;69:41-7.

9. Rossignol DA, Frye RE. Mitochondrial dysfunction in autism spectrum disorders: a systematic review and meta-analysis. Mol Psychiatry. 2012;17:290-314 
10. Lombard J. Autism: a mitochondrial disorder? Med Hypotheses. 1998;50:497-500

11. Valenti D, de Bari L, De Filippis B, Henrion-Caude A, Vacca RA. Mitochondrial dysfunction as a central act or in intellectual disability-related diseases: an overview of Down syndrome, autism, Fragile $X$ and Rett syndrome. Neurosci Behav Rev. 2014;46:202-17.

12. Giulivi C, Zhang YF, Omanska-Klusek A, Ross-Inta C, Wong S, HertzPicciotto l, et al. Mitochondrial dysfunction in autism. JAMA. 2010;304:2389-96.

13. Frye RE. Biomarkers of abnormal energy metabolism in children with autism spectrum disorder. NAJ Med Sci. 2012;5:141-7.

14. Oliveira G, Diogo L, Grazina M, Garcia P, Ataíde A, Marques C, et al. Mitochondrial dysfunction in autism spectrum disorders: a population-based study. Dev Med Child Neurol. 2005;47:185-9.

15. Goldenthal MJ, Damle S, Sheth S, Shah N, Melvin J, Jethva R, et al. Mitochondrial enzyme dysfunction in autism spectrum disorders; a novel biomarker revealed from buccal swab analysis. Biomark Med. 2015;9(10):957-65.

16. Parikshak NN, Gandal MJ, Geschwind DH. Systems biology and gene networks in neurodevelopmental and neurodegenerative disorders. Nat Rev Genet. 2015;16(8):441-58.

17. Napoli E, Wong S, Hertz-Picciotto I, Giulivi C. Deficits in bioenergetics and impaired immune response in granulocytes from children with autism. Pediatrics. 2014;133(5):e1405-10.

18. Weissman JR, Kelley RI, Bauman ML, Cohen BH, Murray KF, Mitchell RL, et al. Mitochondrial disease in autism spectrum disorder patients: a cohort analysis. PLoS ONE. 2008;3(11):e3815

19. Palmieri L, Persico AM. Mitochondrial dysfunction in autism spectrum disorders: cause or effect? Biochim Biophys Acta. 2010;1797(6-7):1130-7.

20. Fillano JJ, Goldenthal MJ, Rhodes CH, Marín-García J. Mitochondrial dysfunction in patients with hypotonia, epilepsy, autism, and developmenta delay: HEADD syndrome. J Child Neurol. 2002;17(6):435-9.

21. Smith M, Spence MA, Flodman P. Nuclear and mitochondrial genome defects in autism. Ann NY Acad Sci. 2009;1151:102-32.

22. Gu F, Chauhan V, Kaur K, Brown WT, LaFauci G, Wegiel J, et al. Alterations in mitochondrial DNA copy number and the activities of electron transport chain complexes and pyruvate dehydrogenase in the frontal cortex from subjects with autism. Transl Psychiatry. 2013;3:e299.

23. Pitceathly RD, Rahman S, Hanna MG. Single deletions in mitochondrial DNA-molecular mechanisms and disease phenotypes in clinical practice. Neuromuscul Disord. 2012;22(7):577-86.

24. Haas RH, Parikh S, Falk MJ, Saneto RP, Wolf NI, Darin N, et al. Mitochondrial disease: a practical approach for primary care physicians. Pediatrics. 2007:120:1326-33.

25. Méhes K. Informative morphogenetic variants (minor congenital anomalies). Orv Hetil. 1986;127(49):3001-3.

26. NEPSYBANK. Magyar Klinikai Neurogenetikai Társaság, Budapest. http:// molneur.webdoktor.hu. Accessed 03 April 2016.

27. Remenyi V, Inczedy-Farkas G, Komlosi K, Horvath R, Maasz A, Janicsek I, et al. Retrospective assessment of the most common mitochondrial DNA mutations in a large Hungarian cohort of suspect mitochondrial cases. Mitochondrial DNA. 2015;26(4):572-8.

28. Betancur C. Etiological heterogeneity in autism spectrum disorders: more than 100 genetic and genomic disorders and still counting. Brain Res. 2011:1380:42-77.

29. Chi square, Fisher exact test. http://vassarstats.net/odds2x2.html. Accessed 20 Jul 2017

30. Picard Tools-By Broad Institute. http://broadinstitute.github.io/picard/. Accessed 02 Jun 2017

31. Li H, Durbin R. Fast and accurate short read alignment with BurrowsWheeler transform. Bioinformatics. 2009;25(14):754-60

32. DePristo MA, Banks E, Poplin R, Garimella KV, Maguire JR, Hartl C, et al. A framework for variation discovery and genotyping using next-generation DNA sequencing data. Nat Genet. 2011:43(5):491-8.

33. Cingolani P, Platts A, le Wang L, Coon M, Nguyen T, Wang L, et al. A program for annotating and predicting the effects of single nucleotide polymorphisms, SnpEff: SNPs in the genome of Drosophila melanogaster strain w1118; iso-2; iso-3. Fly. 2012;6(2):80-92.

34. Balicza P, Grosz Z, Gonzalez MA, Bencsik R, Pentelenyi K, Gal A, et al. Genetic background of the hereditary spastic paraplegia phenotypes in Hungary_an analysis of 58 probands. J Neurol Sci. 2016;364:116-21.
35. Cooper DN, Ball EV, Krawczak M. The human gene mutation database. Nucleic Acids Res. 1998;26(1):285-7.

36. Morava E, van den Heuvel L, Hol F, de Vries MC, Hogeveen M, Rodenburg RJ, et al. Mitochondrial disease criteria: diagnostic applications in children. Neurology. 2006;67(10):1823-6.

37. Taylor RW, Pyle A, Griffin H, Blakely EL, Duff J, He L, et al. Use of wholeexome sequencing to determine the genetic basis of multiple mitochondrial respiratory chain complex deficiencies. JAMA. 2014;312(1):68-77.

38. Richards S, Aziz N, Bale S, Bick D, Das S, Gastier-Foster J, et al. Standards and guidelines for the interpretation of sequence variants: a joint consensus recommendation of the American College of Medical Genetics and Genomics and the Association for molecular pathology. Genet Med. 2015;17(5):405-24.

39. Aramaki M, Udaka T, Kosaki R, Makita Y, Okamoto N, Yoshihashi H, et al. Phenotypic spectrum of CHARGE syndrome with CHD7 mutations. J Pediatr. 2006:148:410-4

40. Karniely S, Weekes MP, Antrobus R, Rorbach J, vanHaute L, Umrania Y, et al. Human cytomegalovirus infection upregulates the mitochondrial transcription and translation machineries. Mbio. 2016;7(2):e00029.

41. Basson MA, van Ravenswaaij-Arts C. Functional insights into chromatin remodelling from studies on CHARGE syndrome. Trends Genet. 2015;31(10):600-11.

42. Koyanagi M, Asahara S, Matsuda T, Hashimoto N, Shingeyama Y, Shibutani $Y$, et al. Ablation of TSC2 enhances insulin secretion by increasing the number of mitochondria through activation of mTORC1. PLOS ONE. 2011;6:e23238

43. Chang S, Ren G, Steiner RD, Merkens LS, Roullet JB, Korade Z. Elevated autophagy and mitochondrial dysfunction in the Smith-Lemli-Opitz syndrome. Mol Genet Metabol Rep. 2014;1:431-42.

44. Kornblum C, Nicholls TJ, Haack TB, Schöler S, Peeva V, Danhauser K, et al. Loss-of-function mutations in MGME1 impair mtDNA replication and cause multisystemic mitochondrial disease. Nat Genet. 2013;45:214-9.

45. Tyynismaa H, Ylikallio E, Patel M, Molnar MJ, Haller RG, Suomalainen A. A heterozygous truncating mutation in RRM2B causes autosomaldominant progressive external ophthalmoplegia with multiple mtDNA deletions. Am J Hum Genet. 2009;5(2):290-5.

46. Suomalianen A, Kaukonen J. Disease caused by nuclear genes affecting mtDNA stability. Am J Meg Genet. 2001;106:53-61.

47. Chalkia D, Singh LN, Leipzig J, Lvova M, Derbeneva O, Lakatos A, et al. Association between mitochondrial DNA Haplogroup variation and autism spectrum disorders. JAMA Psychiatry. 2017;74(11):1161-8.

48. Pentelenyi K, Remenyi V, Gal A, Milley GM, Csosz A, Mende BG, et al. Asian-specific mitochondrial genome polymorphism (9-bp deletion) in Hungarian patients with mitochondrial disease. Mitochondrial DNA A. 2016;27(3):1697-700

49. Beunders G, van de Kamp J, Vasudevan P, Morton J, Smets K, Kleefstra T, et al. A detailed clinical analysis of 13 patients with AUTS2 syndrome further delineates the phenotypic spectrum and underscores the behavioural phenotype. J Med Genet. 2016;53(8):523-32.

50. Bartlett CW, Flax JF, Logue MW, Vieland VJ, Bassett AS, Tallal P, et al. A major susceptibility locus for specific language impairment is located on 13q21. Am J Hum Genet. 2002;71:45-55

51. Bi W, Ohyama T, Nakamura H, Yan J, Visvanathan J, Justice MJ, et al. Inactivation of Rai1 in mice recapitulates phenotypes observed in chromosome engineered mouse models for Smith-Magenis syndrome. Hum Mol Genet. 2005;14:983-95.

52. Zhang F, Potocki L, Sampson JB, Liu P, Sanchez-Valle A, Robbins-Furman $P$, et al. Identification of uncommon recurrent Potocki-Lupski syndromeassociated duplications and the distribution of rearrangement types and mechanisms in PTLS. Am J Hum Genet. 2010;86:462-70.

53. Girirajan S, Elsas LJ, Devriendt K, Elsea SH. RAl1 variations in SmithMagenis syndrome patients without 17p11.2 deletions. J Med Genet. 2005;42:820-8.

54. McDonald ML, MacMullen C, Liu DJ, Leal SM, Davis RL. Genetic association of cyclic AMP signaling genes with bipolar disorder. Transl Psychiatry. 2012;2(10):e169.

55. MitoBreak. http://mitobreak.portugene.com. Accessed 27 Nov 2017.

56. Guo Y, Deng X, Zhang J, Su L, Xu H, Luo Z, et al. Analysis of the MRPL3, DNAJC13 and OFCC1 variants in Chinese Han patients with TS-CTD. Neurosci Lett. 2012;517(1):18-20. 